\title{
Randomized trial of intracardiac echocardiography-guided slow pathway ablation
}

\author{
Peter Kupo ${ }^{1}$ - Laszlo Saghy ${ }^{1}$ - Gabor Bencsik ${ }^{1}$ - Maria Kohari ${ }^{1}$. Attila Makai ${ }^{1}$. Mate Vamos ${ }^{1}$ - Attila Benak ${ }^{1}$. \\ Marton Miklos ${ }^{1} \cdot$ Gabriela Raileanu $^{1} \cdot$ Noemi Schvartz $^{1} \cdot$ Robert Pap $^{1}$
}

Received: 31 October 2021 / Accepted: 13 January 2022

(c) The Author(s), under exclusive licence to Springer Science+Business Media, LLC, part of Springer Nature 2022

\begin{abstract}
Purpose Radiofrequency (RF) catheter ablation of the slow pathway (SP) in atrioventricular nodal reentry tachycardia (AVNRT) is highly effective; however, it may require prolonged fluoroscopy and RF time. We postulated that visualization of the SP region with intracardiac echocardiography (ICE) could decrease ablation time, minimize radiation exposure, and facilitate SP ablation compared to the standard, fluoroscopy-guided approach.

Methods In our study, we randomized 91 patients undergoing electrophysiologic study and SP ablation for AVNRT into 2 groups: fluoroscopy-only $(n=48)$ or ICE-guided $(n=43)$ group. Crossover to ICE-guidance was allowed after 8 unsuccessful RF applications.

Results Mapping plus ablation time (mean \pm standard deviation: $18.8 \pm 16.1 \mathrm{~min}$ vs $11.6 \pm 15.0 \mathrm{~min}, p=0.031$ ), fluoroscopy time (median [interquartile range]: 4.9 [2.93-8.13] $\mathrm{min}$ vs. 1.8 [1.2-2.8] $\mathrm{min}, p<0.001$ ), and total ablation time (144 [104-196] s vs. 81 [60-159] s, $p=0.001)$ were significantly shorter in the ICE group. ICE-guidance was associated with reduced radiation exposure (13.2 [8.2-13.4] mGy vs. 3.7 [1.5-5.8] mGy, $p<0.001)$. The sum of delivered RF energy (3866 [2786-5656] Ws vs. 2283 [1694-4284] Ws, $p=0.002)$ and number of RF applications (8 [4.25-12.75] vs. 4 [2-7], $p=0.001)$ were also lower with ICE-guidance. Twelve (25\%) patients crossed over to the ICE-guided group. All were treated successfully thereafter with similar number, time, and cumulative energy of RF applications compared to the ICE group. No recurrence occurred during the follow-up.

Conclusions ICE-guidance during SP ablation significantly reduces mapping and ablation time, radiation exposure, and RF delivery in comparison to fluoroscopy-only procedures. Moreover, early switching to ICE-guided ablation seems to be an optimal choice in challenging cases.
\end{abstract}

Keywords Atrioventricular nodal reentrant tachycardia $\cdot$ Intracardiac echocardiography $\cdot$ Slow pathway ablation $\cdot$ Radiation exposure $\cdot$ Radiofrequency catheterablation

\section{Introduction}

Atrioventricular (AV) nodal reentrant tachycardia (AVNRT) is the most common form of paroxysmal supraventricular tachycardia [1]. AVNRT represents reentry in the area of the AV node, but the exact circuit remains elusive. Considerable evidence points to the inferior nodal extensions providing the substrate for slow pathway (SP) conduction [1].

Peter Kupo

peter.kupo@gmail.com

1 2nd Department of Internal Medicine and Cardiology Centre, Medical School, University of Szeged, Semmelweis u. 8., H-6725, Szeged, Hungary
Catheter ablation of the SP is considered first-line therapy for AVNRT [2]. This treatment option is curative for patients with AVNRT, and has high acute and long-term success and low recurrence rates [2,3]. Despite a high success rate, ablation of the SP can be challenging in certain cases, likely due to anatomical variations [4]. During SP ablation procedures, the positioning of the ablation catheter is guided anatomically with attention to intracardiac electrograms [5-8]. For anatomical orientation, fluoroscopy and/or three-dimensional (3D) electroanatomical mapping system (EAMS) are used. However, these methods do not allow direct visualization of intracardiac structures, therefore are less helpful for SP ablation in patients with atypical anatomy [9]. 
Intracardiac echocardiography (ICE) is a unique, realtime imaging technique to assist catheter ablations by visualizing intracardiac structures, ablation catheter position, stability, and lesion [10, 11].

We hypothesized that real-time, direct visualization of the SP region using ICE can identify anatomical variations and facilitate the ablation procedure. The aim of this study was to compare conventional, fluoroscopy-guided versus ICEguided SP ablation in consecutive patients with AVNRT.

\section{Methods}

\subsection{Study population}

In our prospective singe-center study, 91 consecutive patients undergoing electrophysiologic study and SP radiofrequency (RF) ablation for AVNRT were randomized into fluoroscopy-guided or ICE-guided groups. We excluded patients referred for a second (redo) procedure, those in whom other arrhythmias were also identified, and patients under 18 years of age. The protocol of the study is in accordance with the Declaration of Helsinki and the study protocol was approved by the regional ethics committee (Approval no.: 4649). All patients provided written informed consent for the study protocol.

\subsection{Study protocol}

Antiarrhythmic drugs were discontinued for at least five half-lives prior to the procedure. The electrophysiologic (EP) study was performed under conscious sedation using midazolam \pm fentanyl, in a fasting state. After local anesthesia, following femoral venous access, one decapolar steerable catheter (interelectrode spacing 2-5-2 mm, Dynamic Deca, Bard Electrophysiology, Lowell, MA, USA) was positioned in the coronary sinus (CS), one quadripolar electrode catheter in the right ventricular apex (RVA), and one mapping/ablation catheter was positioned in the His bundle region. Twelve lead electrocardiogram (ECG) and intracardiac electrograms were recorded and stored on a digital recording system (CardioLab, GE Healthcare, Chicago, IL, USA), using a band pass filter between 30 and $500 \mathrm{~Hz}$. Atrioventricular nodal conduction and the induction of AVNRT were tested by using electrical stimulation techniques. The $\mathrm{S} 2$ coupling interval was shortened by $10 \mathrm{~ms}$ after each drive-train until tachycardia was induced, or AV conduction block occurred, or the atrial refractory period was reached. If the tachycardia was not inducible, isoprenaline infusion $(1-2 \mu \mathrm{g} / \mathrm{min})$ was administered to increase the heart rate by at least $20 \%$, and the same stimulation protocol was repeated during infusion and during the washout phase.
The diagnosis of AVNRT was based on established electrophysiologic criteria and pacing maneuvers including an A- $(\mathrm{H})-\mathrm{V}$ response after ventricular overdrive pacing, with an SA-VA interval $>85 \mathrm{~ms}$ and corrected postpacing interval minus tachycardia cycle length $>110 \mathrm{~ms}$ [12].

After the diagnostic EP study, in patients randomized to the ICE-group, the RVA quadripolar electrode catheter was removed and an $8 \mathrm{~F}$ ICE catheter (AcuNaVTM $90 \mathrm{~cm}$, Siemens Medical Solutions, Mountain View, CA, USA) was inserted to guide mapping and SP ablation. The echotransducer was positioned in the low right atrium at the 6 o'clock position and rotated clockwise towards the septum. The imaging plane allowed the visualization of anatomic landmarks such as the septal cavotricuspid isthmus, ostium of the coronary sinus, tricuspid valve, right ventricle, and aortic root. The RF ablation catheter (CELSIUS ${ }^{\circledR}$ Catheter, Biosense Webster, CA, USA) was then placed between the coronary sinus ostium and the tricuspid ring guided by ICE (Fig. 1). The proximity of the ablation catheter to the compact AV node was judged by the distance from the aortic valve, which invariably marks the recording site of a proximal His potential. RF energy was delivered in sinus rhythm starting just below the CS with a power output of $30 \mathrm{~W}$ and a preset temperature of $55^{\circ} \mathrm{C}$. Applications were considered effective when junctional rhythm appeared. Effective applications were continued for 30 to $60 \mathrm{~s}$. RF application delivery was immediately discontinued in the case of catheter displacement, sudden impedance rise, PR interval prolongation, anterograde AV, or retrograde VA block. In case of ineffective RF delivery, the ablation catheter was moved slightly closer to the aortic valve (but never closer than $0.5 \mathrm{~cm}$ ) and RF application was attempted again.

In the conventional arm, catheter positioning was guided by fluoroscopic landmarks provided by the EP catheters and aiming at an atrial-to-ventricular electrogram amplitude ratio of 1:3-1:5. Crossover from the fluoroscopy-guided group to an ICE-guided procedure was allowed when after $8 \mathrm{RF}$ applications, the ablation endpoint was not reached.

The procedural success of the ablation was obtained if the arrhythmia could not be induced after a 20-min waiting period and if there were no observations of $>1$ echo beats, on and off isoprenaline.

Procedure time (in minutes) was measured from the time point of first femoral puncture until the withdrawal of the last venous sheath. Mapping plus ablation time was measured from the beginning of the mapping for the SP to the end of the last ablation attempt. Fluoroscopy time (in minutes), radiation dose (in $\mathrm{mGy}$ ), and dose-area product (DAP in $\mathrm{Gycm}^{2}$ ) were automatically recorded by the fluoroscopy system. Ablation data (total number of the RF applications, sum of delivered RF energy expressed in Ws, and the total ablation time expressed in seconds) were calculated and stored by the EP recording system (CardioLab, GE Healthcare). 
Fig. 1 Direct visualization of the ablation catheter in the slow pathway region by intracardiac echocardiography (ICE). Abbreviations: Abl, ablation catheter; Ao, aortic root; RA, right atrium; RV, right ventricle

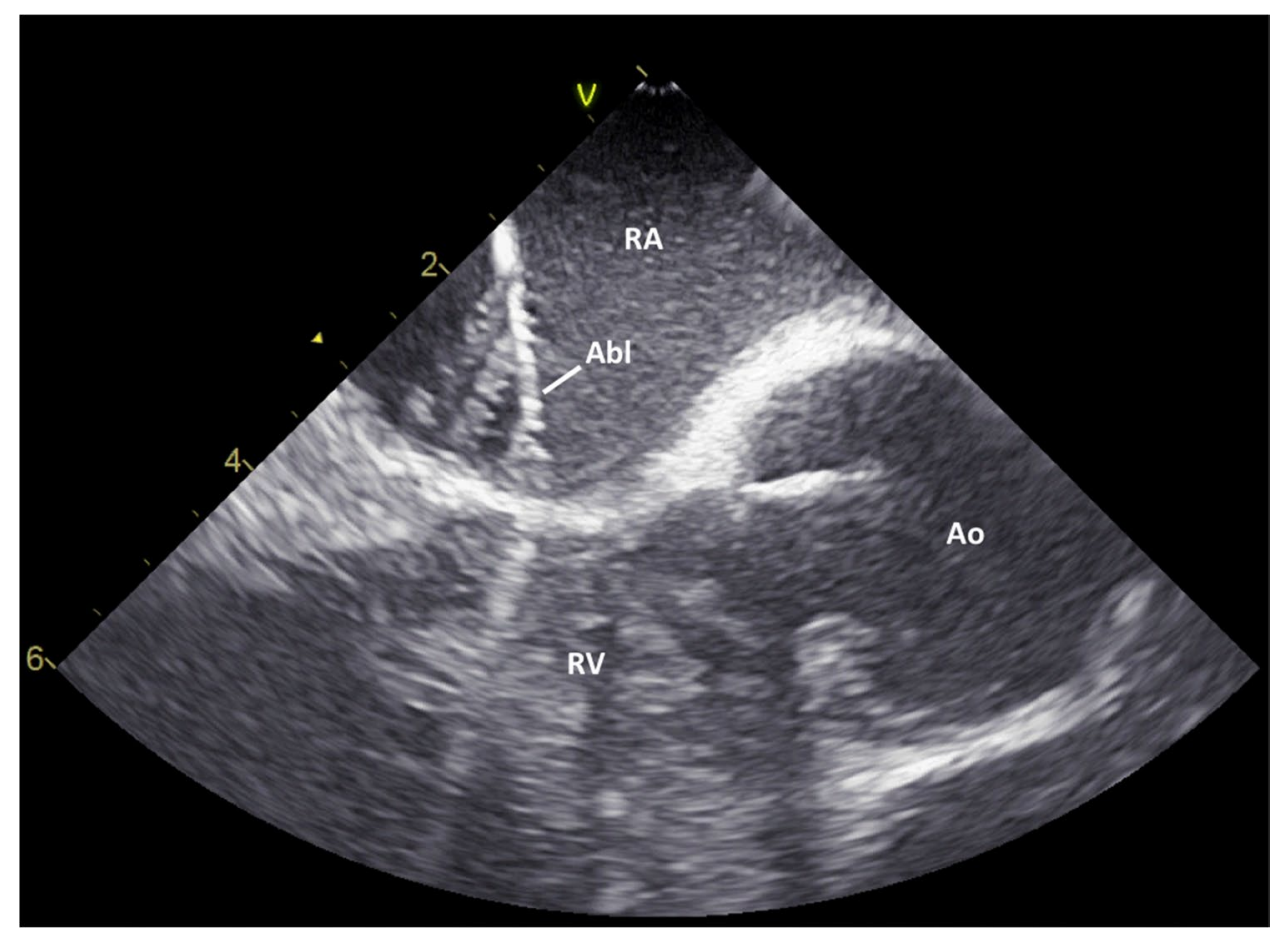

\subsection{Statistical analysis}

The distribution pattern of the data was evaluated using the Shapiro-Wilk tests. All tests were performed two-tailed with a significance level set to $p<0.05$. Continuous data were presented as the mean $\pm \mathrm{SD}$ or median (interquartile range, IQR), as appropriate while categorical variables are presented as absolute numbers and percentages. For comparisons, chi-square test and Mann-Whitney $U$ test were used as appropriate. Statistical analyses were performed using SPSS 24 software (SPSS, Inc., Chicago, IL, USA).

\section{Results}

A total of 91 patients were randomized in 2 groups: 48 patients to the fluoroscopy-only and 43 patients to the ICEguided group. There was no difference regarding the baseline characteristics of the study patients between the groups (male sex: $40.4 \%$ vs. $28.6 \%, p=0.27$; age: $53.4 \pm 13.8 \mathrm{~min}$ vs. $51.0 \pm 17.2$ years, $p=0.49$ ).

The procedural endpoint was achieved in all 91 cases; thus, acute success rate was $100 \%$. We found no difference in puncture to mapping time between fluoroscopy-only vs. ICE-guided groups $(43 \pm 16.8 \mathrm{~min}$ vs. $42.8 \pm 16.1 \mathrm{~min}$, $p=0.95)$. Mapping plus ablation time $(18.8 \pm 16.1 \mathrm{~min}$ vs. $11.6 \pm 15.0 \mathrm{~min}, p=0.031)$, fluoroscopy time $(4.9$ (2.93-8.13) min vs. $1.8(1.2-2.8) \mathrm{min}, p<0.001)$, and total ablation time (144 (104-196) s vs. $81(60-159) \mathrm{s}, p=0.001)$ were significantly shorter in the ICE-guided group (Fig. 2).
ICE-guidance was associated with reduced fluoroscopy dose (13.2 (8.2-13.4) mGy vs. 3.7 (1.5-5.8) mGy, $p<0.001)$ and radiation exposure $\left(0.99(0.56-1.57) \mathrm{Gycm}^{2}\right.$ vs. 0.22 $\left.(0.14-0.51) \mathrm{Gycm}^{2}, p<0.001\right)$.

The sum of delivered RF energy (3866 (2786-5656) Ws vs. 2283 (1694-4284) Ws, $p=0.002)$ and number of RF applications (8 (4.25-12.75) vs. $4(2-7), p=0.001)$ were also lower with ICE-guidance (Table 1).

Out of 48 patients randomized to the fluoroscopy-only group, twelve patients (25\%) crossed over to the ICE-guided group. All were treated successfully thereafter and the number, time, and cumulative energy of RF applications delivered after crossover were similar to those recorded in the ICE-guided group (Table 2).

There was no complication including AV block during the study. No recurrence occurred during the $14.8 \pm 2.9$-month follow-up.

\section{Discussion}

We found in this randomized comparison of fluoroscopyversus ICE-guided ablation of the SP for AVNRT that ICE use is beneficial in reducing mapping plus ablation time, radiation exposure, and unnecessary RF delivery. It also facilitates treatment of challenging cases.

Supported by its low complication and a high success rate, catheter ablation is the treatment of choice for paroxysmal supraventricular tachyarrhythmias including AVNRTs [2, 3]. Katritsis et al. have shown that ablation 

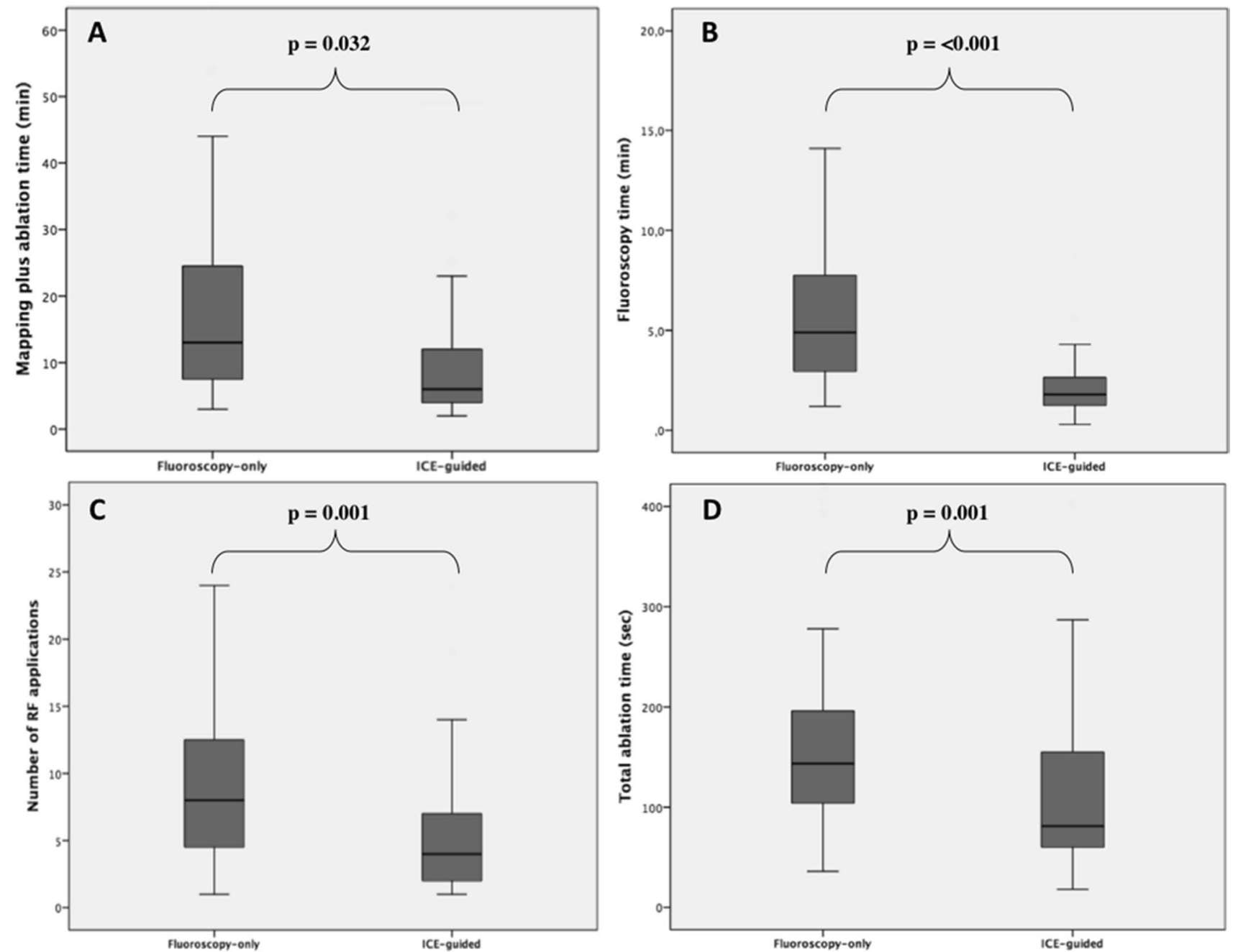

Fig. 2 Box plots presenting mapping plus ablation time (A), fluoroscopy time (B), number of RF applications (C), and RFA time (D) in the fluoroscopy-only and the ICE-group. Abbreviations: ICE, intracardiac echocardiography; RF, radiofrequency; RFA, radiofrequency ablation

Table 1 Procedural parameters in the study population

\begin{tabular}{lllr}
\hline & Fluoroscopy-only & ICE-guided & $p$ value \\
\hline Puncture to mapping time (min) & $43 \pm 16.8$ & $42.8 \pm 16.1$ & 0.95 \\
Mapping plus ablation time (min) & $18.8 \pm 16.1$ & $11.6 \pm 15.0$ & 0.032 \\
Fluoroscopy time (min) & $4.9(2.93-8.13)$ & $1.8(1.2-2.8)$ & $<0.001$ \\
Fluoroscopy dose (mGy) & $13.18(8.23-23.37)$ & $3.68(1.49-5.76)$ & $<0.001$ \\
Radiation exposure (Gycm $\left.{ }^{2}\right)$ & $0.99(0.56-1.57)$ & $0.22(0.14-0.51)$ & $<0.001$ \\
Total ablation time (s) & $144(104-196)$ & $81(60-159)$ & 0.001 \\
Number of RF applications & $8(4.25-12.75)$ & $4(2-7)$ & 0.001 \\
Sum of delivered RF energy (Ws) & $3866(2786-5656)$ & $2283(1694-4284)$ & 0.002 \\
\hline
\end{tabular}

$I C E$, intracardiac echocardiography; $R F$, radiofrequency at the anatomical area of the slow pathway (SP) is the therapy of choice for symptomatic AVNRT, regardless of whether the typical or atypical form is present [13]. The contemporary anatomical approach for SP ablation targets the lower below the CS ostium [14, 15], traditionally using fluoroscopy-guidance, often with prolonged X-ray and ablation times $[9,14]$. This exposes both patients and medical staff to a potentially dangerous amount of ionizing radiation and results in unnecessary RF delivery to the heart in proximity to the conduction system, with a 
Table 2 Procedural parameters in the crossover group

\begin{tabular}{ll}
\hline & Crossover group \\
\hline Fluoroscopy time from CO $(\mathrm{min})$ & $0.05(0-0.55)$ \\
Fluoroscopy dose from CO $(\mathrm{mGy})$ & $0.16(0.01-1.1)$ \\
Radiation exposure from CO $\left(\mathrm{Gycm}{ }^{2}\right)$ & $0.01(0-0.14)$ \\
Ablation time from CO $(\mathrm{s})$ & $67(49-262)$ \\
Number of RF applications from CO & $5.5(2.5-8.75)$ \\
Sum of delivered RF energy from CO (Ws) & $2405(1263-4624)$ \\
\hline
\end{tabular}

$C O$, crossover; $R F$, radiofrequency

possible association with late $\mathrm{AV}$ nodal conduction disturbances [16].

Koch's triangle varies in terms of anatomy and electrogram distribution (Supplementary videos 1 and 2) [4, 7]. ICE allows direct real-time visualization of the relevant anatomical structures resulting in more precise and targeted mapping and ablation. Bencsik et al. previously demonstrated that ICE-guided ablation of the cavotricuspid isthmus significantly shortens the procedure and fluoroscopy time and time spent for ablation in comparison with fluoroscopy-only procedures without an EAMS [17]. Previously studies reported on the advantage of mechanical radial ICEimaging for SP ablation $[18,19]$. Most recently, data from a retrospective analysis of 47 patients who underwent ICE vs. fluoroscopy-guided SP cryoablation showed that the use of ICE shortens the cryo-application duration; however, time needed for catheter placement is longer, when compared with conventional fluoroscopic guidance [20].

Recently the 3D mapping system-guided approach for catheter ablation of simple arrhythmias like AVNRT has become widespread [21-23]. However, while EAMSs can significantly reduce fluoroscopy time and dose, they may be less helpful in detecting variations in anatomy due to the lack of direct visualization of anatomical structures. Therefore, they may not provide benefit in terms of the number and cumulative time of RF ablations. This was shown in a meta-analysis published in 2016 comparing randomized controlled trials of zero/near zero-fluoroscopy versus conventionally performed procedures, and there was no difference in either total ablation time or procedure time between the groups [24]. Subsequent trials also did not show difference either regarding ablation time or application number [25-27]. One study showed increased procedure time with the use of an EAMS in AVNRT without benefit in success or complications [9].

These studies, however, did not use contact force sensing technology that could be similar to ICE in detecting contact between the ablation catheter and atrial tissue.

Compared to the above-mentioned studies on EAMS in AVNRT ablation, our study has shown that using ICE-guidance during RF ablation of the SP not only reduces radiation exposure, but also minimizes unnecessary RF delivery by direct visualization of both the ablation catheter and the SP region. Reduced RF energy delivery may impact the development of late conduction disturbances after SP ablation [28, 29].

Remarkably, in challenging fluoroscopy-guided procedures, after crossover to ICE-guidance similar number, time and cumulative energy of RF applications were needed as in the original ICE-guided group. This finding suggests that SP ablation in case of unusual anatomy is just as straightforward when the target area and catheter are directly visualized by ICE.

The only drawback of an ICE-guided strategy to SP ablation can be the extra cost of the catheter. Compared to the extra cost of using an EAMS, this expenditure is similar [30], or even lower if using reprocessed ICE catheters [31]. Compared to an EAMS, ICE offers the potential added advantage of a better targeted ablation with less energy delivered and facilitation of treatment of more challenging cases.

\subsection{Limitation}

Our series is a single center study, which may limit its applicability in other centers. Although the study was randomized, it could not be blinded. The level of ICE experience varied among the investigators. As discussed above, the extra cost of using an ICE catheter cannot be neglected. It also requires a larger venous sheath; however, no increase in access site complications occurred due to this. Multicenter trials are required to assess the cost-effectiveness and ability to improve clinical outcomes with this technique.

\section{Conclusion}

Compared to conventional fluoroscopy-guided procedures, ICE-guidance for anatomic SP ablation significantly reduces mapping and ablation time, radiation exposure, and RF delivery. When conventional ablation is ineffective after a fair effort, switching to ICE-guided ablation can be recommended.

Supplementary Information The online version contains supplementary material available at https://doi.org/10.1007/s10840-022-01126-y.

\section{Declarations}

Conflict of interest The authors declare no competing interests.

\section{References}

1. Katritsis DG, Camm AJ. Atrioventricular nodal reentrant tachycardia. Circulation 122:831-840. https://doi.org/10.1161/CIRCU LATIONAHA.110.936591

2. Brugada J, Katritsis DG, Arbelo E, et al. 2019 ESC guidelines for themanagement of patients with supraventricular tachycardia. Eur Heart J. 2020;41(5):655-720. https://doi.org/10.1093/eurhe artj/ehz467. 
3. Katritsis DG, Boriani G, Cosio FG, et al. European Heart Rhythm Association (EHRA) consensus document on the management of supraventricular arrhythmias, endorsed by Heart Rhythm Society (HRS), Asia-Pacific Heart Rhythm Society (APHRS), and Sociedad Latinoamericana de Estimulación Cardiaca y Elect. Eur Heart J. 2017;19(3):465-511. https://doi.org/10.1093/europace/euw301.

4. Inoue S, Becker AE. Koch's triangle sized up: anatomical landmarks in perspective of catheter ablation procedures. PACE - Pacing Clin Electrophysiol. 1998;21(8):1553-8. https://doi.org/10. 1111/j.1540-8159.1998.tb00242.x.

5. Jackman WM, Beckman KJ, McClelland JH, et al. Treatment of supraventricular tachycardia due to atrioventricular nodal reentry by radiofrequency catheter ablation of slow-pathway conduction. N Engl J Med. 1992;327(5):313-8. https://doi.org/10.1056/nejm1 99207303270504.

6. Wathen M, Natale A, Wolfe K, et al. An anatomically guided approach to atrioventricular node slow pathway ablation. Am J Cardiol. 1992;70(9):886-9. https://doi.org/10.1016/00029149(92)90732-E.

7. Yamaguchi T, Tsuchiya T, Nagamoto Y, et al. Anatomical and electrophysiological variations of Koch's triangle and the impact on the slow pathway ablation in patients with atrioventricular nodal reentrant tachycardia: a study using 3D mapping. J Interv Card Electrophysiol. 2013;37(1):111-20. https://doi.org/10.1007/ s10840-012-9769-z.

8. Bhaskaran A, Albarri M, Ross N, et al. Slow pathway radiofrequency ablation using magnetic navigation: a description of technique and retrospective case analysis. Hear Lung Circ. 2017;26(12):1297-302. https://doi.org/10.1016/j.hlc.2017.01.007.

9. Chrispin J, Misra S, Marine JE, et al. Current management and clinical outcomes for catheter ablation of atrioventricular nodal re-entrant tachycardia. Europace. 2018;20(4):e51-9. https://doi. org/10.1093/europace/eux110.

10. Chu E, Fitzpatrick AP, Chin MC, et al. Radiofrequency catheter ablation guided by intracardiac echocardiography. Circulation. 1994;89(3):1301-5. https://doi.org/10.1161/01.CIR.89.3.1301.

11. Stellbrink C, Siebels J, Hebe J, et al. Potential of intracardiac ultrasonography as an adjunct for mapping and ablation. Am Heart J. 1994;127(4):1095-101. https://doi.org/10.1016/00028703(94)90094-9.

12. Katritsis DG, Josephson ME. Differential diagnosis of regular, narrow-QRS tachycardias. Heart Rhythm. 2015;12(7):1667-76. https://doi.org/10.1016/j.hrthm.2015.03.046.

13. Katritsis DG, Marine JE, Contreras FM, et al. Catheter ablation of atypical atrioventricular nodal reentrant tachycardia. Circulation. 2016;134:1655-63. https://doi.org/10.1161/CIRCULATIO NAHA.116.024471.

14. Katritsis DG, Zografos T, Siontis KC, et al. Endpoints for successful slow pathway catheter ablation in typical and atypical atrioventricular nodal re-entrant tachycardia: a contemporary, multicenter study. JACC Clin Electrophysiol. 2019;5:113-9. https://doi.org/ 10.1016/j.jacep.2018.09.012.

15. Katritsis DG, Josephson ME. Classification of electrophysiological types of atrioventricular nodal re-entrant tachycardia: a reappraisal. Europace. 2013;15(9):1231-40.

16. Kesek M, Lindmark D, Rashid A, Jensen SM. Increased risk of late pacemaker implantation after ablation for atrioventricular nodal reentry tachycardia: a 10-year follow-up of a nationwide cohort. Heart Rhythm. 2019;16(8):1182-8. https://doi.org/10. 1016/j.hrthm.2019.02.032.

17. Bencsik G, Pap R, Makai A, et al. Randomized trial of intracardiac echocardiography during cavotricuspid isthmus ablation. $\mathrm{J}$ Cardiovasc Electrophysiol. 2012;23(9):996-1000. https://doi.org/ 10.1111/j.1540-8167.2012.02331.x.

18. Fisher WG, Pelini MA, Bacon ME. Adjunctive intracardiac echocardiography to guide slow pathway ablation in human atrioventricular nodal reentrant tachycardia: anatomic insights. Circulation. 1997;96(9):3021-9. https://doi.org/10.1161/01.CIR. 96.9.3021.

19. Batra R, Nair M, Kumar M, et al. Intracardiac echocardiography guided radiofrequency catheter ablation of the slow pathway in atrioventricular nodal reentrant tachycardia. J Interv Card Electrophysiol. 2002;6(1):43-9. https://doi.org/10.1023/A:1014124206 594.

20. Luani B, Rauwolf T, Genz C, et al. Intracardiac echocardiography versus fluoroscopy for endovascular and endocardial catheter navigation during cryo-ablation of the slow pathway in AVNRT patients. Cardiovasc Ultrasound. 2019;17:12. https://doi.org/10. 1186/s12947-019-0162-2.

21. Gaita F, Guerra PG, Battaglia A, Anselmino M. The dream of near-zero X-rays ablation comes true. Eur Heart J. 2016;37(36):2749-55. https://doi.org/10.1093/eurheartj/ehw223.

22. Casella M, Dello Russo A, Pelargonio G, et al. Near zero fluoroscopic exposure during catheter ablation of supraventricular arrhythmias: the NO-PARTY multicentre randomized trial. Europace. 2016;18(10):1565-72. https://doi.org/10.1093/europ ace/euv344.

23. Nagaraju L, Menon D, Aziz PF. Use of 3D electroanatomical navigation (CARTO-3) to minimize or eliminate fluoroscopy use in the ablation of pediatric supraventricular tachyarrhythmias. PACE - Pacing Clin Electrophysiol. 2016;39(6):574-80. https://doi.org/ $10.1111 /$ pace. 12830 .

24. Yang L, Sun G, Chen X, et al. Meta-analysis of zero or nearzero fluoroscopy use during ablation of cardiac arrhythmias. Am J Cardiol. 2016;118(10):1511-8. https://doi.org/10.1016/j.amjca rd.2016.08.014

25. Swissa M, Birk E, Dagan T, et al. Radiofrequency catheter ablation of atrioventricular node reentrant tachycardia in children with limited fluoroscopy. Int J Cardiol. 2017;236:198-202. https://doi. org/10.1016/j.ijcard.2017.01.128.

26. Walsh KA, Galvin J, Keaney J, et al. First experience with zerofluoroscopic ablation for supraventricular tachycardias using a novel impedance and magnetic-field-based mapping system. 2018;107:578-585. https://doi.org/10.1007/s00392-018-1220-8

27. Di Cori A, Zucchelli G, Segreti L, et al. Predictors of zero $\mathrm{X}$ ray procedures in supraventricular arrhythmias ablation. 2020;36(9):1599-1607. https://doi.org/10.1007/ s10554-020-01884-8

28. Decroocq M, Rousselet L, Riant M, et al. Periprocedural, early, and long-Term risks of pacemaker implantation after atrioventricular nodal re-entry tachycardia ablation: a French nationwide cohort. Europace. 2020;22(10):1526-36. https://doi.org/10.1093/ europace/euaa151.

29. Liao JN, Hu YF, Wu TJ, et al. Permanent pacemaker implantation for late atrioventricular block in patients receiving catheter ablation for atrioventricular nodal reentrant tachycardia. Am J Cardiol. 2013;111(4):569-73. https://doi.org/10.1016/j.amjcard.2012.11. 003.

30. Winkle RA, Mead RH, Engel G, et al. Physician-controlled costs: the choice of equipment used for atrial fibrillation ablation. J Interv Card Electrophysiol 36:157-165. https://doi.org/10.1007/ s10840-013-9782-x

31. Bank AJ, Berry JM, Wilson RF, Lester BR. Acceptance criteria for reprocessed AcuNav ${ }^{\circledR}$ catheters: comparison between functionality testing and clinical image assessment. Ultrasound Med Biol. https://doi.org/10.1016/j.ultrasmedbio.2008.09.013

Publisher's note Springer Nature remains neutral with regard to jurisdictional claims in published maps and institutional affiliations. 